Article

\title{
Preparation and Preliminary Evaluation of Povidone Single-Chain Nanoparticles as Potential Drug Delivery Nanocarriers
}

\author{
Isabel Asenjo-Sanz ${ }^{1}$, Maite Del-Corte ${ }^{1}$, Jokin Pinacho-Olaciregui ${ }^{1}$, Marina \\ González-Burgos ${ }^{1}$, Edurne González ${ }^{1}$, Ester Verde-Sesto ${ }^{1}$, Arantxa Arbe ${ }^{1}$, \\ Juan Colmenero ${ }^{1,2,3}$, José A. Pomposo ${ }^{1,2,4, *}$ \\ 1 Centro de Física de Materiales (CSIC, UPV/EHU) and Materials Physics Center \\ MPC, Paseo Manuel de Lardizabal 5, E-20018 San Sebastián, Spain \\ 2 Departamento de Física de Materiales, Universidad del País Vasco (UPV/EHU), \\ Apartado 1072, E-20800 San Sebastián, Spain \\ 3 Donostia International Physics Center (DIPC), Paseo Manuel de Lardizabal 4, \\ E-20018 San Sebastián, Spain \\ 4 IKERBASQUE-Basque Foundation for Science, María Díaz de Haro 3, E-48013 \\ Bilbao, Spain \\ * Correspondence: José A. Pomposo, Email: Josetxo.pomposo@ehu.eus.
}

\section{ABSTRACT}

One of the key areas in nanomedicine is the use of nanometer-sized materials as nanocarriers for therapeutic and diagnostic (theranostic) purposes. In particular, nanoparticles (NPs) have attracted a considerable attention due to their small size that confers the ability to be transported more easily through the body. Ideally, nanocarriers would be biocompatible and biodegradable so the involvement of soft matter-based NPs is an interesting approach. Folding individual polymer chains to single-chain nanoparticles (SCNPs) endows the resulting soft nano-objects with promising prospects for drug encapsulation and subsequent controlled delivery. In this work, we report on the preparation and preliminary (in vitro) evaluation of Povidone SCNPs as potential drug delivery nanocarriers. We select Povidone (polyvinylpyrrolidone) as a water-soluble polymer with a large

\section{G Open Access}

Received: 23 May 2019

Accepted: 08 July 2019

Published: 10 July 2019

Copyright (c) 2019 by the author(s). Licensee Hapres, London, United Kingdom. This is an open access article distributed under the terms and conditions of Creative Commons Attribution 4.0 International License. commercial use in medicine, which is biocompatible and non-antigenic as well as safe for oral and topical applications. For evaluation of Povidone SCNPs as drug delivery nanocarriers, we select two drugs with reported anti-cancer activity: (i) Cisplatin, a widely used hydrophilic anticancer agent for treatment of a variety of cancer cells; and (ii) Lovastatin, a lipophilic compound with in vitro anti-proliferative, pro-apoptotic and anti-invasive effects in different cancer cell lines. After showing release of these drugs from Povidone SCNPs, we demonstrate that these nanoparticles can be rendered fluorescent in combination with functional aggregation-induced emission (AIE) fluorophore molecules paving the way to the potential development of theranostic Povidone SCNPs. 
KEYWORDS: Povidone; single-chain nanoparticles; drug delivery; nanomedicine

\section{ABBREVIATIONS}

EC, European Commission; NPs, nanoparticles; SCNPs, single-chain nanoparticles; CNTs, carbon nanotubes; QD, quantum dots; MRI, magnetic resonance imaging; CT, computed tomography; PET, positron-emission tomography; PVP, polyvinylpyrrolidone; ACQ, aggregation-caused quenching; AIE, aggregation-induced emission; SEC, size exclusion chromatography; DLS, dynamic light scattering; SAXS, small angle X-ray scattering; UV-Vis, ultraviolet-visible; PL, photoluminescence; CP, Cisplatin; LO, Lovastatin; TPE-BA, [(1,2-Diphenylethene-1,2-diyl)bis-(4,1-phenylene)]diboronic acid

\section{INTRODUCTION}

According to the European Commission (EC) recommendation 2011/696/EU, nanomaterial is defined as a natural, incidental or manufactured material containing particles, in an unbound state or as an aggregate or as an agglomerate and where, for $50 \%$ or more of the particles in the number size distribution, one or more external dimensions is in the size range of $1 \mathrm{~nm}$ to $100 \mathrm{~nm}\left(1 \mathrm{~nm}=0.001 \mu \mathrm{m}=10^{-9} \mathrm{~m}\right)$ [1]. Nanoparticles (NPs) have attracted a considerable attention due to their small size that confers the ability to be transported more easily through the body [2]. Several types of nanoparticles have been synthesized in recent years: (i) metal-based NPs (e.g., gold NPs, gold nano-rods, gold nano-shells, super-paramagnetic iron oxide NPs) [3], (ii) carbon-based NPs (e.g., graphene NPs, single-wall carbon nanotubes (CNTs) and multi-wall CNTs) [4], (iii) semiconductor-based NPs (cadmium selenide quantum dots (QDs), cadmium telluride QDs) [5], and (iv) soft matter-based NPs, including single-chain nanoparticles (SCNPs) formed via folding of individual polymer chains [6], among other ones. Each specific composition endows these NPs with characteristic electrical, optical, magnetic (etc.) properties for different specific purposes.

Concerning the use of NPs in nanomedicine, the biological transport performed anatomically into cellular and sub-cellular levels is severely affected by NP's physical and chemical attributes [7]. In this way, NPs have to overcome several obstacles/barriers ( $\mathrm{pH}$, cell membrane, pores, chemical or enzymatic reactions...) to reach their target. One of the key areas in nanomedicine is the use of nanometer-sized materials as nanocarriers for diagnostic and therapeutic (theranostic) purposes [8]. NPs' physical and chemical attributes including size, shape, flexibility, hydrophobicity, etc., are critical for a successful theranostic achievement. One of the critical points to overcome when drug target is located intracellularly is drug passage through the cell membrane [9]. Phagocytation happens when macrophage ingests large nanoparticles 
between 0.25 to $3 \mu \mathrm{m}$ that have been previously a foreign body [10]. Non-phagocytic clathrin-mediated endocytosis is another way of internalization, which may be performed by ligands that are bound to nanoparticle receptor and an invagination process happens to introduce NPs with a size between 120 and $200 \mathrm{~nm}$ [11]. Finally, nanoparticles can also be introduced into the cellular compartment by calveolae-mediated endocytosis, i.e., through invaginations present in membrane by pores below $80 \mathrm{~nm}$ [12].

Ideally, nanocarriers would be biocompatible, biodegradable, and specifically designed to obtain an appropriate bio-distribution. Researchers have been able to synthesize a variety of NPs loaded with drugs that can be observed by imaging methods (e.g., magnetic resonance imaging (MRI) [13], computed tomography (CT) [14], positron emission tomography (PET) [15], fluorescence microscopy [16]). Among the potential advantages that could be achieved using NPs loaded with therapeutic molecules we can mention: (i) improved physical and chemical stability of drug; (ii) increased absorption of drugs; (iii) controlled release of drugs; (iv) superior crossing of tissue/cell-specific barriers to penetrate into cells; and (v) reduced adverse effects and toxicity associated with the administration of free drugs. However, the main concern of NPs in nanomedicine is their potential toxicity for living systems, mainly derived from their accumulation in different tissues and organs [17]. In this way, using soft matter-based nanocarriers seems to be a particularly interesting solution.

In this work, we report on the preparation and preliminary (in vitro) evaluation of Povidone single-chain nanoparticles (SCNPs) as potential drug delivery nanocarriers (see Figure 1). Polyvinylpyrrolidone, also known as Povidone or PVP, is a highly polar, amphoteric polymer used in the pharmaceutical field as an excipient, as well as in the cosmetic, food and textile industries [18]. PVP is biocompatible and non-antigenic, safe for oral and topical applications, and it is easily dispersible in water. Its conjugation with iodine forms the active ingredient PVP-iodine, largely used as antiseptic (e.g., Betadine). We hypothesized that by folding individual PVP chains at high dilution via intra-chain cross-linking [19], the resulting Povidone SCNPs will be endowed with local domains (pockets) to which drugs can be temporary immobilized for their subsequent controlled delivery. Previously, we and others have demonstrated the possibilities that SCNPs offer for the construction of responsive drug delivery nanocarriers [20-22]. A detailed analysis of recent findings in the field of drug delivery from SCNPs can be found in a relevant review paper by Kröger and Paulusse [23]. 


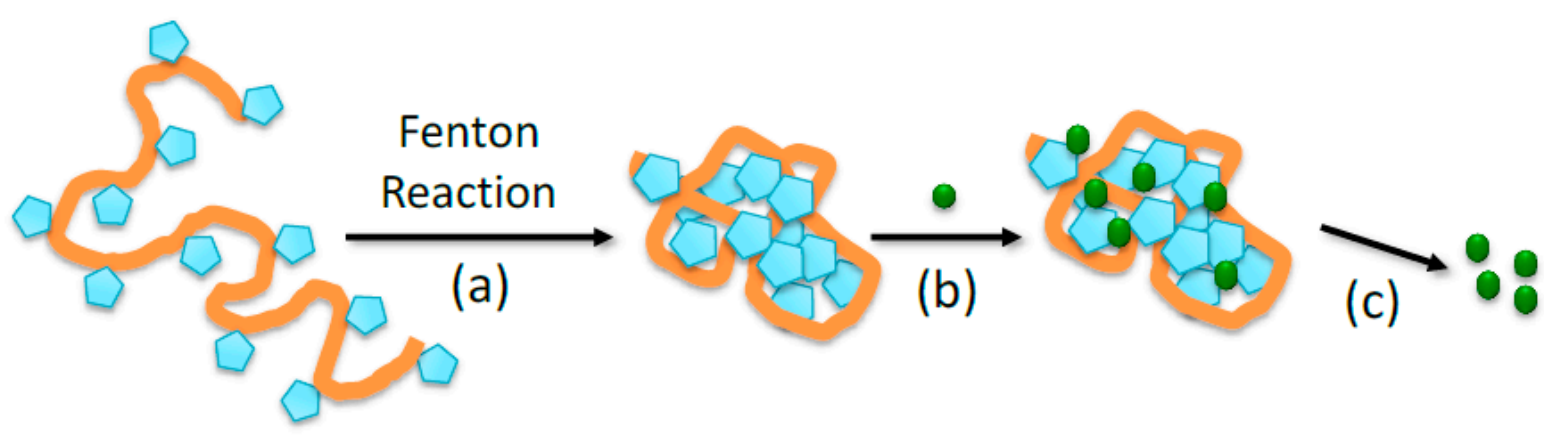

Figure 1. (a) Folding of individual linear Povidone chains to Povidone single-chain nanoparticles (SCNPs) through radical generation in the pyrrolidone units via Fenton reaction followed by intra-chain radical-radical coupling events. (b) Schematic illustration of drug encapsulation by Povidone SCNPs as nanocarriers. (c) Schematic illustration of controlled drug delivery from drug-loaded Povidone SCNPs.

Following a previous recent investigation [24], we will perform the folding of PVP chains through radical generation in the pyrrolidone units via Fenton reaction followed by intra-chain radical-radical coupling events. For evaluation of Povidone SCNPs as potential drug delivery nanocarriers, we selected two drugs with reported anti-cancer activity [25,26]: (i) Cisplatin, a widely used hydrophilic anticancer agent that has been shown to be highly effective in the treatment of testicular, ovarian, breast, bladder, lung, head and neck cancers; and (ii) Lovastatin, a lipophilic compound which has demonstrated to exert anti-proliferative, pro-apoptotic and anti-invasive effects in different cancer cell lines with varying sensitivity. After showing the drug delivery characteristics of Povidone SCNPs as nanocarriers, we will turn our attention to the possibility to render fluorescent the Povidone SCNPs. Toward this end, we select a new kind of fluorophore molecules which are opposite to conventional ones, typically affected by the well-known and deleterious aggregation-caused quenching (ACQ) effect [27]. Hence, we investigated the use of a functional aggregation-induced emission (AIE) fluorophore [28] to introduce diagnostic characteristics into the Povidone SCNPs.

\section{MATERIALS AND METHODS}

\section{Materials}

Povidone (polyvinylpyrrolidone, PVP, homopolymer of molecular weight $360 \mathrm{kDa}$, China), hydrogen peroxide solution $\left(\mathrm{H}_{2} \mathrm{O}_{2}, 30\right.$ wt \% in $\mathrm{H}_{2} \mathrm{O}$, ACS reagent, USA), iron (II) chloride $\left(\mathrm{FeCl}_{2}, 98 \%\right.$, USA), acetic acid (ACS reagent, $\geq 99.8 \%$, USA), chloroform $\left(\mathrm{CHCl}_{3}, \geq 99.8 \%\right.$, USA), $N, N$-dimethylformamide (DMF for molecular biology, $\geq 99 \%$, USA), dimethyl sulfoxide (DMSO, $\geq 99.5 \%$, USA), cis-diamineplatinum(II) dichloride (Cisplatin, CP, European Pharmacopoeia Reference Standard, USA), [(1S,3R,7S,8S,8a R)-8-[2-[(2R,4R)-4-hydroxy-6-oxooxan-2-yl]ethyl]-3,7-dimet hyl-1,2,3,7,8,8a-hexahy-dronaphthalen-1-yl](2S)-2-methylbutanoate (Lovastatin, LO, European Pharmacopoeia Reference Standard, USA), [(1,2-Diphenylethene-1,2-diyl)bis-(4,1-phenylene)] diboronic acid (TPE-BA, 
$96 \%$, USA) and dialysis tubing cellulose membrane (averaged flat width $76 \mathrm{~mm}$, typical molecular weight cut-off: $14 \mathrm{kDa}$, Germany) were purchased from Aldrich and used, unless specified, as received. Sodium chloride (NaCl, puriss., for HPLC) was purchased from Fluka (Spain). For synthesis, deionized water obtained from a Thermo Scientific apparatus (Barnstead TII Pure Water System, USA) was employed. For SEC, water (HPLC grade, Aldrich, Switzerland), sodium azide $\left(\mathrm{NaN}_{3}, \geq 99.5 \%\right.$, Aldrich, USA), sodium nitrate $\left(\mathrm{NaNO}_{3}\right.$, extra pure, Scharlab, Spain) and sodium phosphate monobasic (anhydrous, $\mathrm{NaH}_{2} \mathrm{PO}_{4}, \geq 99.0 \%$, Fluka, Germany) were used.

\section{Characterization Techniques}

\section{Size exclusion chromatography}

Size exclusion chromatography (SEC) measurements were performed at $35{ }^{\circ} \mathrm{C}$ on a triple-detection Agilent PL-GPC 50 system (UK) with Agilent light scattering, refractive index and viscosimetry detectors (UK), equipped with PL aquagel-OH Guard $(8 \mu \mathrm{m})$ and PL aquagel-OH MIXED-H $(8 \mu \mathrm{m})$ columns (Agilent, UK). Data analysis was performed with the Agilent SEC software. HPLC water, $0.02 \mathrm{M} \mathrm{NaNO}_{3}, 0.01 \mathrm{M} \mathrm{NaH}_{2} \mathrm{PO}_{4}$, $0.02 \mathrm{wt} \% \mathrm{NaN}_{3}$ (filtered to $0.2 \mu \mathrm{m}, \mathrm{pH} \approx 7$ ) was used as eluent at a flow rate of $1 \mathrm{~mL} / \mathrm{min}$.

\section{Dynamic light scattering}

A Malvern Zetasizer Nano ZS apparatus (Malvern Instruments, UK) was used to determine the hydrodynamic radius of the samples in deionized water at $25^{\circ} \mathrm{C}$.

\section{Small-angle X-ray scattering}

Small-angle X-ray scattering (SAXS) experiments were conducted on Rigaku 3-pinhole PSAXS-L equipment (Rigaku, USA) operating at $45 \mathrm{kV}$ and $0.88 \mathrm{~mA}$. The MicroMax-002+ X-ray generator system (Rigaku, USA) is composed by a microfocus sealed tube source module and an integrated X-ray generator unit which produces $\mathrm{Cu} \mathrm{Ka}$ transition photons of wavelength $\lambda=1.54 \AA$. The scattered X-rays are detected on a two-dimensional multi-wire X-ray detector (Gabriel design, 2D-200X, USA) and converted to one-dimensional scattering curves by radial averaging. This gas-filled proportional type detector offers a $200 \mathrm{~mm}$ diameter active area with $c a .200 \mu \mathrm{m}$ resolution. After radial integration, the scattered intensities were obtained as a function of momentum transfer $Q=4 \pi \lambda^{-1}$ $\sin \theta$, where $\theta$ is half the scattering angle. Reciprocal space calibration was done using silver behenate as standard. The sample-to-detector distance was $2 \mathrm{~m}$, covering a Q-range between 0.01 and $0.20 \AA^{-1}$. The measurements were performed at room temperature in capillaries of $2 \mathrm{~mm}$ thickness. The data were corrected for background scattering due 
to capillaries and solvent. Scattering cross sections were obtained in absolute units by using water as calibration standard.

\section{Ultraviolet-visible spectroscopy}

Ultraviolet-visible (UV-Vis) spectroscopy measurements were performed at $25{ }^{\circ} \mathrm{C}$ in an Agilent 8453A apparatus (China) with Peltier thermostatic cell holder, T-controller 89090A (Agilent, China).

\section{Fluorescence spectroscopy}

Photoluminiscence (PL) spectra were recorded at room temperature on an Agilent Cary Eclipse spectrometer (USA) at an excitation wavelength of $365 \mathrm{~nm}$.

\section{Synthesis of Povidone Single-Chain Nanoparticles (SCNPs)}

Povidone SCNPs were synthesized following the method reported in [24]. In brief, PVP-based SCNPs were produced at room temperature by mixing two solutions: solution A containing Povidone dissolved in water (50 mg, $100 \mathrm{~mL}, 0.5 \mathrm{mg} / \mathrm{mL}$ ) and $\mathrm{H}_{2} \mathrm{O}_{2}(113 \mu \mathrm{L}, 10 \mathrm{mM}$ ), and solution $\mathrm{B}$ containing Povidone dissolved in water (same concentration and amount as solution A) and $\mathrm{FeCl}_{2}(63.4 \mathrm{mg}, 5 \mathrm{mM}$ ). The $\mathrm{pH}$ of the mixed system was adjusted at $\mathrm{pH}=3.5$ with acetic acid $(0.1 \mathrm{M})$. After reaction completion, the mixture was dialyzed for $24 \mathrm{~h}$ in order to remove traces of unreacted $\mathrm{FeCl}_{2}$. Finally, the system was freeze-dried and Povidone SCNPs were obtained as white powders.

\section{Preparation of Cisplatin-Loaded Povidone SCNPs}

First, a solution of Cisplatin (CP) in water ( $5 \mu \mathrm{g} / \mathrm{mL}$, containing $0.9 \%$ of $\mathrm{NaCl}$ ) was prepared. Then, $1.5 \mathrm{mg}$ of Povidone SCNPs were dissolved in $5 \mathrm{~mL}$ of the solution containing $\mathrm{CP}$ and the mixture was incubated for $24 \mathrm{~h}$ in the dark at room temperature. In order to purify the CP-loaded Povidone nanocarriers, extraction with $5 \mathrm{~mL}$ of $\mathrm{CHCl}_{3}$ was carried out (this process was repeated twice); $\mathrm{CHCl}_{3}$ was evaporated using a continuous argon stream, and the CP-loaded Povidone nanocarriers were isolated.

\section{Cisplatin Drug Delivery from Povidone SCNPs}

The delivery of cisplatin from CP-loaded Povidone SCNPs was determined by UV-Vis spectroscopy measurements at $265 \mathrm{~nm}$. To quantify the in vitro release of $\mathrm{CP}$ from the Povidone nanocarriers, samples were collected periodically for $96 \mathrm{~h}$. After a given time $t$, the absorbance of the sample was recorded at $265 \mathrm{~nm}$ and the released $\mathrm{CP}$ from the nanocarriers was determined by means of the following equation:

$$
\text { CP Released }(\%)=\left[\left(\mathrm{CP}_{t}-\mathrm{CP}_{t=0}\right) /\left(\mathrm{CP}_{t=96 \mathrm{~h}}-\mathrm{CP}_{t=0}\right)\right] \times 100
$$


where $\mathrm{CP}_{t}$ is the value of absorbance at $265 \mathrm{~nm}$ measured at time $t(\mathrm{~h})$, $\mathrm{CP}_{t=0}$ is the value of absorbance measured at the beginning of the experiment, and $\mathrm{CP}_{t=96 \mathrm{~h}}$ is the value of absorbance measured after $96 \mathrm{~h}$.

\section{Preparation of Lovastatin-Loaded Povidone SCNPs}

A solution of Lovastatin (LO) $(5 \mu \mathrm{g} / \mathrm{mL})$ in a 1:1 mixture of DMF and water was prepared. Then, $1.5 \mathrm{mg}$ of Povidone SCNPs were dissolved in $5 \mathrm{~mL}$ of the solution containing $\mathrm{LO}$ and the mixture was incubated for $24 \mathrm{~h}$ at room temperature. The resulting LO-loaded Povidone SCNPs were purified by dialysis against pure water using dialysis tubing cellulose membrane with a molecular weight cut-off of $14 \mathrm{kDa}$. Upon dialysis for 3 days, all the excess amount of LO was found to precipitate, so the resulting water solution containing the LO-loaded Povidone SCNPs was filtered, and the system was freeze-dried to isolate the LO-loaded nanocarriers.

\section{Lovastatin Drug Delivery from Povidone SCNPs}

The delivery of Lovastatin from the single-chain PVP nanocarriers was determined by UV-Vis spectroscopy measurements at $288 \mathrm{~nm}$. To quantify the in vitro release of LO from the LO-loaded nanocarriers, samples were periodically collected for $2.5 \mathrm{~h}$. After a given time $t$, the absorbance of each sample was recorded at $288 \mathrm{~nm}$ and the released LO from the nanocarriers was determined by means of the following equation:

$$
\text { LO Released (\%) }=\left[\left(\mathrm{LO}_{t}-\mathrm{LO}_{t=0}\right) /\left(\mathrm{LO}_{t=2.5 \mathrm{~h}}-\mathrm{LO}_{t=0}\right)\right] \times 100
$$

where $\mathrm{LO}_{t}$ is the value of absorbance at $288 \mathrm{~nm}$ measured at time $t(\mathrm{~h})$, $\mathrm{LO}_{t=0}$ is the value of absorbance measured at the beginning of the experiment, and $\mathrm{LO}_{t=2.5 \mathrm{~h}}$ is the value of absorbance measured after $2.5 \mathrm{~h}$.

\section{Preparation of Fluorescent Povidone SCNPs}

Povidone SCNPs were dissolved in water at a concentration of $1 \mathrm{mg} / \mathrm{mL}$. To $900 \mu \mathrm{L}$ of this solution, $100 \mu \mathrm{L}$ of a stock solution composed of TPE-BA $0.5 \mathrm{mM}$ in DMSO were added under stirring. Upon addition of TPE-BA, fluorescence was observed by the naked eye using a portable laboratory UV-lamp (Spectroline, USA).

\section{RESULTS AND DISCUSSION}

\section{Preparation and Size Characterization of Povidone SCNPs}

Following a recent investigation [24], we performed the folding of Povidone chains through radical generation in the pyrrolidone units via Fenton reaction followed by intra-chain radical-radical coupling events (see Figure 1 and ref. [24] for details). The synthesis process was performed by starting from a commercially available water-soluble PVP homopolymer and by using reactive oxygen species (ROS) generated via 
Fenton reaction for the intra-chain folding/collapse process of individual polymer chains.

Hydroxyl radical is the most reactive radical among the reactive oxygen species. Its reaction with Povidone is expected to generate macroradicals centered in three possible positions, taking into account the labiality of the hydrogen atoms present in its structure (see Figure 2) [29]. In general, the rate of hydrogen abstraction is dependent on the dissociation energy of the $\mathrm{X}-\mathrm{H}$ bond to form the radical. Basic thermodynamic calculations show that $\mathrm{C}-\mathrm{H}$ bonds a-positioned to a heteroatom or a $\mathrm{C}=\mathrm{O}$ group are lower in energy, mainly due to the stabilization of the radical product [30]. Within the three $\mathrm{C}-\mathrm{H}$ bonds shown in Figure 2, the most favorable PVP macro-radical expected via Fenton reaction is highlighted in green in this figure. Then, Povidone SCNPs would be formed by working at high dilution conditions via intra-chain coupling of these radicals leading to the formation of covalent $\mathrm{C}-\mathrm{C}$ bonds. It is worth mentioning that an insoluble Povidone gel (instead of individual Povidone SCNPs) is obtained by using a concentrated solution of neat Povidone (i.e., well above the overlap concentration of Povidone linear chains in solution) instead of a highly diluted Povidone solution.

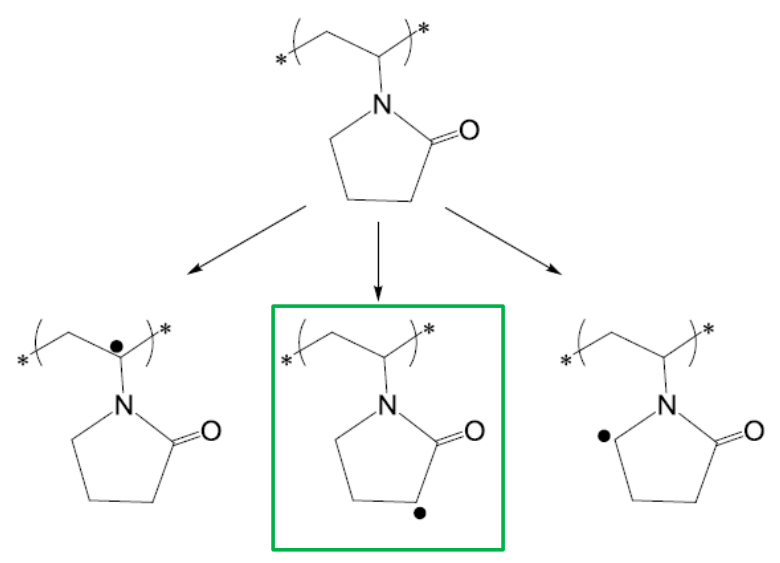

Figure 2. Different macroradicals that can be formed via Fenton reaction [29] (the most favorable one is highlighted in green). Intra-chain radical-radical coupling events are utilized in this work for the formation of Povidone SCNPs.

Experimentally, the intra-chain cross-linking of the individual (linear) Povidone chains to single-chain nanoparticles was performed in water, at room temperature in the presence of hydroxyl radicals (generated by Fenton reaction) and at very dilute conditions $(0.5 \mathrm{mg} / \mathrm{mL})$ in order to avoid inter-molecular cross-linking events. Figure 3 illustrates the significant chain compaction observed by means of SEC and DLS measurements upon formation of Povidone SCNPs. Data about the apparent molecular weight $\left(M_{\mathrm{app}}\right)$ and hydrodynamic radius $\left(R_{\mathrm{H}}\right)$ of Povidone single-chain nanoparticles after $24 \mathrm{~h}$ of reaction are provided in Table 1. 

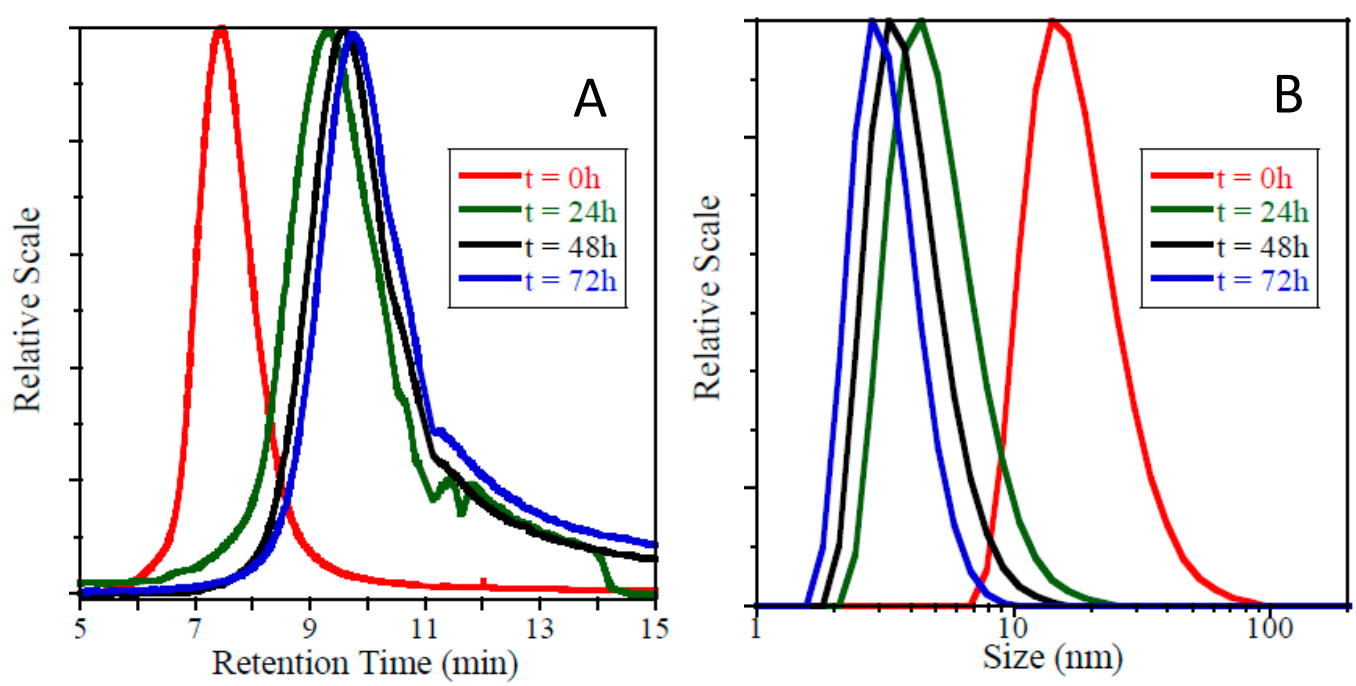

Figure 3. (A) SEC traces showing the progressive increase in peak retention time (i.e., reduction in average hydrodynamic size) during formation of Povidone SCNPs via Fenton reaction. (B) Evolution of the hydrodynamic size distribution as a function of reaction time during Povidone single-chain nanoparticle preparation, as determined by DLS measurements.

Table 1. Apparent molecular weight $\left(M_{\text {app }}\right)$ and hydrodynamic radius $\left(R_{\mathrm{H}}\right)$ of Povidone SCNPs after $24 \mathrm{~h}$ of reaction time.

\begin{tabular}{lll}
\hline $\begin{array}{l}\text { Reaction } \\
\text { Time (h) }\end{array}$ & $\begin{array}{l}\boldsymbol{M}_{\text {app }}{ }^{1} \\
(\text { KDa) }\end{array}$ & $\begin{array}{l}\boldsymbol{R}_{\mathbf{H}} \\
(\mathbf{n m})\end{array}$ \\
\hline 0 & 360.2 & 18.9 \\
24 & 14.7 & 5.3 \\
\hline
\end{tabular}

${ }^{1}$ For definition of $M_{\text {app }}$, see ref. [31].

Complementary SAXS experiments were performed to determine the form factor, radius of gyration $\left(R_{\mathrm{g}}\right)$ and scaling exponent $(\mathrm{U})$ of neat Povidone and the Povidone SCNPs synthesized after $24 \mathrm{~h}$ of reaction time (see Figure 4). Under the high dilution conditions employed, the intermolecular interactions are negligible so the scattered intensity $I(Q)$ in the SAXS experiment directly reflects the form factor $P(Q)$ which provides full information about macromolecular size and shape [32]. Thus, the chain dimensions-quantified via the average radius of gyration $R_{\mathrm{g}}$ as well as the degree of compaction revealed by the scaling exponent $v$ that determines how the chain size depends on the number of monomers $\left(R \propto N^{v}\right)$-can be obtained from the experimental results shown in Figure 4. Quantitatively, the generalized Gaussian coil function allows for a description of the form factor for different values of the scaling exponent [33]. The scaling exponent of Povidone chains in $\mathrm{H}_{2} \mathrm{O}$ obtained in this way takes a value very near to the Flory exponent $\left(U_{\mathrm{F}}=0.59\right)$ corresponding to chains in good solvent conditions with self-avoiding walk conformations. The radius of gyration, $R_{\mathrm{g}}$, decreases from $19.2 \mathrm{~nm}$ (neat Povidone) to $7.2 \mathrm{~nm}$, and the scaling exponent becomes $v=0.55$ for the Povidone SCNPs. Note that this value of scaling 
exponent is larger than that expected for a globular morphology $\left(v_{g}=1 / 3\right)$ or a dense spherical globule with inner bulk density $\left(v_{\mathrm{dsg}}=1 / 4\right)[34]$.
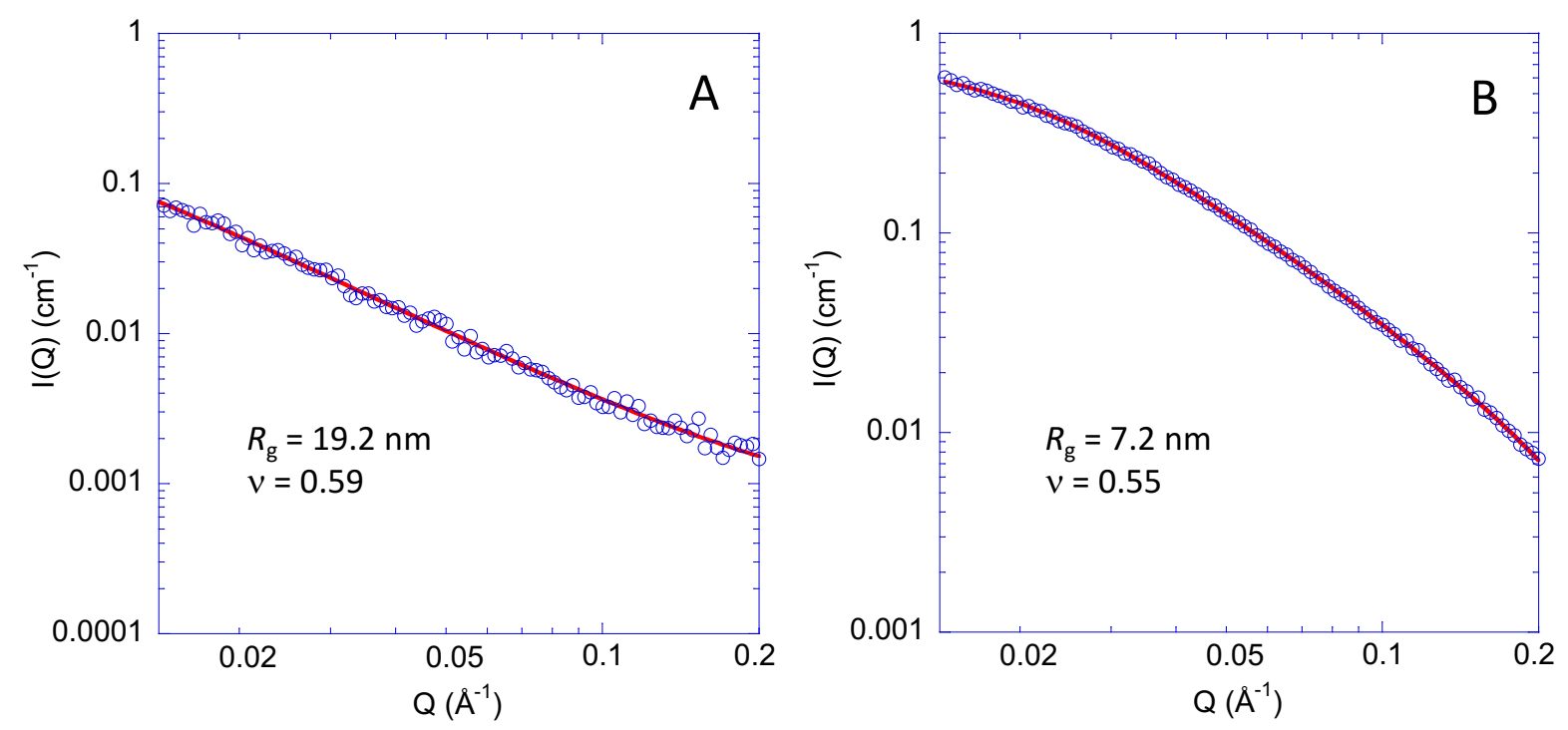

Figure 4. Macromolecular form factors obtained by SAXS experiments on water solutions of Povidone (A) at $4 \mathrm{mg} / \mathrm{mL}$ and the corresponding Povidone SCNPs at $2 \mathrm{mg} / \mathrm{mL}$ obtained by Fenton reaction after $24 \mathrm{~h}$ of reaction time (B). Red lines are fits to generalized Gaussian form factors from which the corresponding values of radius of gyration $\left(R_{\mathrm{g}}\right)$ and scaling exponent $(\mathrm{v})$ are obtained (see text). The higher intensity of the Povidone SCNPs (B) when compared to neat Povidone (A) can be tentatively attributed to the presence of residual iron atoms in the SCNPs, which produce a strong scattering contrast against the solvent.

Taken together, the above SEC, DLS and SAXS data confirm the significant chain compaction that takes place upon folding/collapse of individual Povidone linear chains to SCNPs by means of radical generation in the pyrrolidone units via Fenton reaction followed by intra-chain radical-radical coupling events. We hypothesized that the resulting Povidone SCNPs will be endowed with local domains (pockets) to which drugs can be temporary immobilized for their subsequent controlled delivery [35].

\section{Evaluation of Povidone SCNPs as Potential Drug Delivery Nanocarriers}

For evaluation of Povidone SCNPs as potential drug delivery nanocarriers, we selected two drugs with reported anti-cancer activity: (i) Cisplatin, a widely used hydrophilic anticancer agent for treatment of a variety of cancer cells [24]; and (ii) Lovastatin, a lipophilic compound with in vitro anti-proliferative, pro-apoptotic and anti-invasive effects in different cancer cell lines [26].

\section{Cisplatin $(C P)$}

Povidone SCNPs were loaded with Cisplatin following the procedure described in Materials and Methods section. The size and stability characteristics of the drug-loaded Povidone SCNPs were found to remain 
essentially identical to those of the neat Povidone SCNPs (i.e., hydrodynamic diameter $\approx 11 \pm 3 \mathrm{~nm}$; $\zeta$-potential $\approx-0.025 \pm 0.03 \mathrm{mV}$ ). Figure 5A shows the controlled delivery of CP from Povidone SCNPs to water as monitored by UV-Vis spectroscopy for a period of $96 \mathrm{~h}$. Over time, a clear UV-Vis absorption band develops centered at $\sim 265 \mathrm{~nm}$ which is due to the progressive release of Cisplatin to the water solution. The corresponding in vitro drug delivery curve obtained from the data reported in Figure 5A is illustrated in Figure 5B. These results indicate that CP-loaded Povidone SCNPs have a great potential as nanocarriers since they provide with a sustained, controlled release of Cisplatin that amounts to $\sim 28 \%$ in $2 \mathrm{~h}, \sim 75 \%$ in $24 \mathrm{~h}$, and $~ 95 \%$ in $48 \mathrm{~h}$. These results are in agreement with previous studies concerning the ability of SCNPs to function as drug delivery agents [20-22]. Nevertheless, before practical use of CP-loaded Povidone SCNPs a more complete characterization that is out of the scope of the present paper should be performed (e.g., accurate determination of drug loading content and encapsulation efficiency, evaluation of toxicity issues, effect of $\mathrm{pH}$ and temperature).
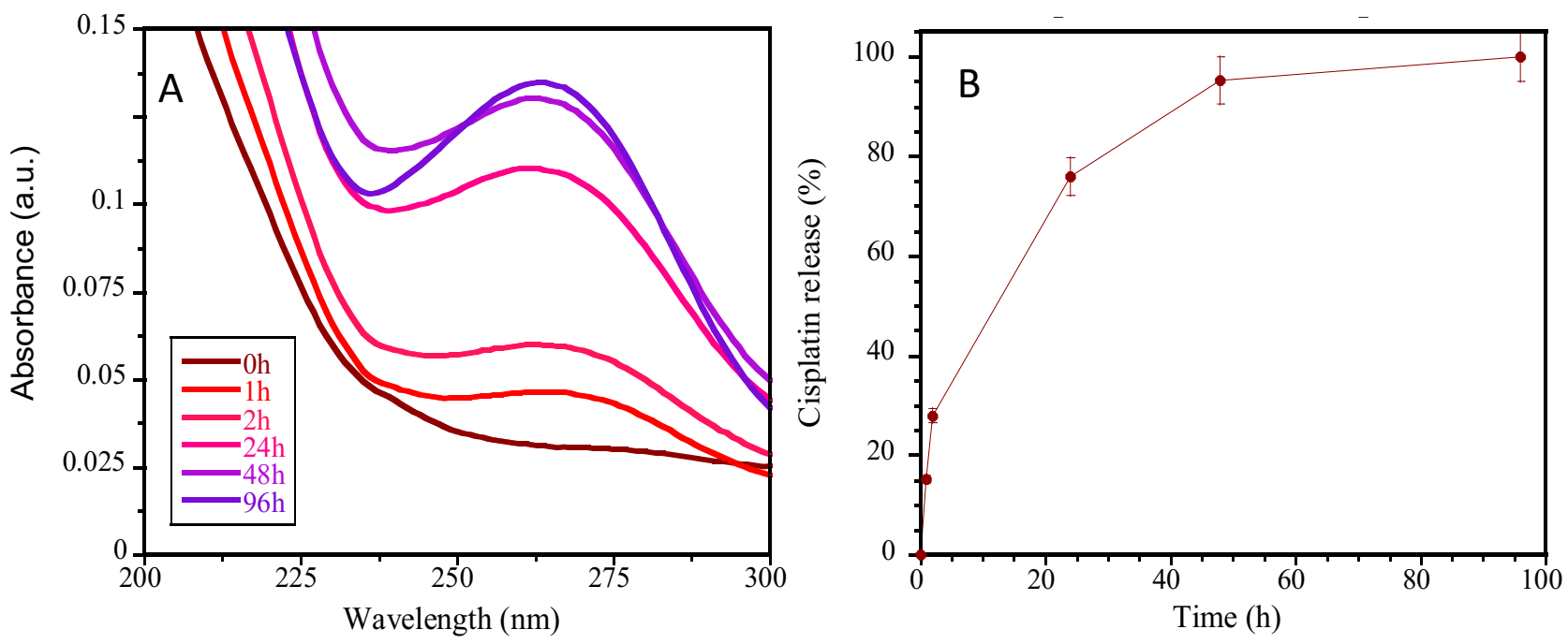

Figure 5. (A) Controlled release of Cisplatin to water from Povidone-loaded SCNPs as monitored by UV-Vis spectroscopy. (B) Drug delivery curve obtained from the evolution over time of the UV-Vis absorption band centered at $265 \mathrm{~nm}$ in (A).

\section{Lovastatin (LO)}

Povidone SCNPs were loaded with Lovastatin following the procedure described in Materials and Methods section. Since LO, which is a lipophilic compound, is almost insoluble in water we monitor the release of this drug to a solvent in which LO is highly soluble, such as DMF. This is a very simple model of LO delivery to lipophilic tissues. Figure $6 \mathrm{~A}$ shows the delivery of Lovastatin from Povidone SCNPs to DMF as monitored by UV-Vis spectroscopy. Over time, a clear UV-Vis absorption band develops centered at $\sim 288 \mathrm{~nm}$ which we attributed to the progressive release of LO to the DMF solution. The corresponding drug delivery curve obtained 
from the data reported in Figure 6A is illustrated in Figure 6B. These results indicate that Povidone SCNPs can be also used as nanocarriers of lipophilic drugs since they provide with a relatively fast release of Lovastatin to DMF that amounts to $63 \%$ in $1 \mathrm{~h}$, and near $100 \%$ in $2 \mathrm{~h}$. However, more realistic models are required to validate these preliminary results, including cellular and toxicity studies as well as a complete characterization of drug loading content and encapsulation efficiency.
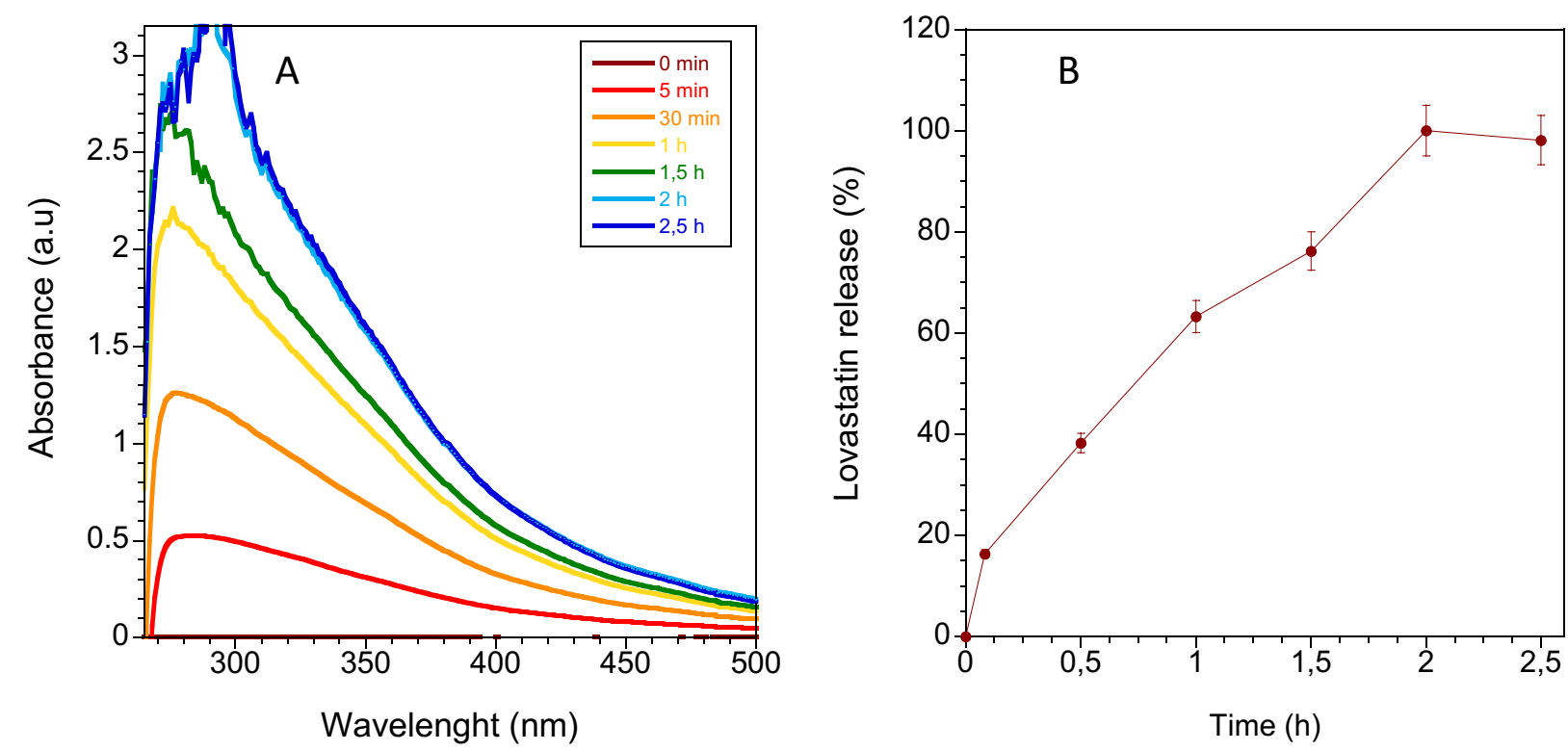

Figure 6. (A) Release of Lovastatin to DMF from Povidone-loaded SCNPs as monitored by UV-Vis spectroscopy. (B) Drug delivery curve obtained from the evolution over time of the UV-Vis absorption band centered at $\sim 288 \mathrm{~nm}$ in (A).

\section{Endowing Povidone SCNPs with Fluorescent Properties}

Fluorophore molecules can be monitored by fluorescence spectroscopy and microscopy, so they have long been used in biomarker analysis, immunoassays, and diagnostic imaging, including cancer diagnosis [36]. However, one of the main limitations of conventional fluorophore molecules is which often suffer from the well-known (and deleterious) aggregation-caused quenching (ACQ) effect [27]. In recent years, a new class of molecules (aggregation-induced emission (AIE) fluorophores) has been discovered that are nearly non-emissive molecules when molecularly dissolved in a solvent but become highly fluorescent when aggregate or after being molecular immobilized due to restriction of the intramolecular rotation in the aggregate/immobilized state leading the excitations to decay radiatively [37,38]. In this work, we evaluate the ability of an AIE fluorophore (TPE-BA, see the chemical structure in Figure 7A) to endow Povidone SCNPs with fluorescent properties. As illustrated in Figure 7B, a water solution of Povidone SCNPs shows almost no fluorescence, as determined by PL measurements. Conversely, a water solution containing neat TPE-BA molecules shows a certain level of fluorescence due to the insolubility of this AIE 
fluorophore under such conditions. In this sense, DLS measurements showed the presence of aggregates with an average size of $R_{\mathrm{H}}=278 \mathrm{~nm}$. Interestingly, a water solution containing both Povidone SCNPs and TPE-BA molecules shows a 5-fold increase in fluorescence (see Figure 7B) and a 13-fold decrease in average aggregate size $\left(R_{\mathrm{H}}=21.3 \mathrm{~nm}\right)$, as determined by DLS measurements. Taken together, the PL and DLS results show the synergistic possibilities offered by the combination of AIE molecules and SCNPs to enhance fluorescence while promoting a small nanoparticle size.
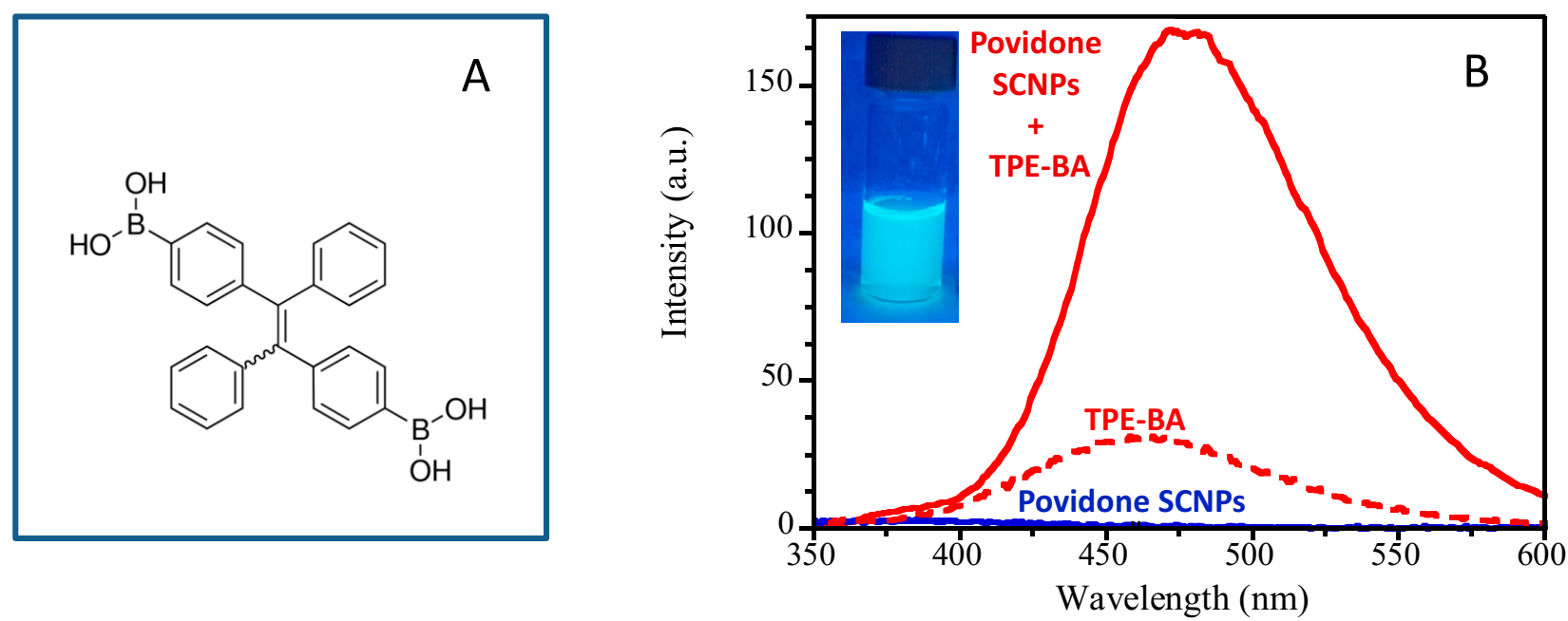

Figure 7. (A) Chemical structure of [(1,2-Diphenylethene-1,2-diyl)bis-(4,1-phenylene)]diboronic acid (TPE-BA) employed in this work as aggregation-induced emission (AIE) fluorophore. (B) PL spectra of Povidone SCNPs (blue trace), TPE-BA (dotted red trace) and Povidone SCNPs + TPE-BA (continuous red trace).

\section{CONCLUSIONS}

In summary, a strategy has been developed toward theranostic SCNPs based on Povidone, a water-soluble polymer with a large commercial use in medicine, which is biocompatible and non-antigenic as well as safe for oral and topical applications. Povidone SCNPs were prepared at high dilution through radical generation in the pyrrolidone units via Fenton reaction followed by intra-chain radical-radical coupling events. Combined SEC, DLS and SAXS data confirmed a significant chain compaction of neat Povidone upon SCNP formation. The resulting Povidone SCNPs were investigated as nanocarriers of two drugs with reported anti-cancer activity and opposite hydrophilic/lipophilic balance: Cisplatin and Lovastatin. The in vitro drug delivery curves of Cisplatin and Lovastatin from Povidone SCNPs have been determined based on UV-Vis measurements. The relative amount of Cisplatin released to water was $\sim 28 \%$ in $2 \mathrm{~h}, \sim 75 \%$ in $24 \mathrm{~h}$, and $\sim 95 \%$ in $48 \mathrm{~h}$, whereas the relative amount of Lovastatin released to DMF was $63 \%$ in $1 \mathrm{~h}$, and near $100 \%$ in $2 \mathrm{~h}$. Moreover, we have shown that Povidone SCNPs can be rendered highly fluorescent by combination with AIE fluorophore molecules, such as TPE-BA. The results from this work are a first step towards the design 
of innovative theranostic nanocarriers based on a cheap, commercially available, water-soluble polymer such as Povidone which is largely used in the pharmaceutical field. Further work will be devoted to determine the drug loading content and encapsulation efficiency of the drug-loaded Povidone SCNPs, their toxicity and $\mathrm{pH} /$ temperature-responsive behaviors, their morphology characterization and stability, as well as to perform a systematic comparison with the neat Povidone homopolymer.

\section{AUTHOR CONTRIBUTIONS}

IAS, MDC, JPO, MGB, EG and EVS contribute to the preparation of Povidone SCNPs, their characterization, and preliminary evaluation as drug delivery nanocarriers. AA and JC performed the analysis of SAXS experiments. JAP conceived the main idea, analyzed the results and wrote the paper with input from all authors.

\section{CONFLICTS OF INTEREST}

The authors declare that there is no conflict of interest.

\section{FUNDING}

This research was funded by Gipuzkoako Foru Aldundia (Programa Red Gipuzkoana de Ciencia, Tecnología e Innovación 2017), grant number RED 101/17, Basque Government, grant number IT-1175-19, and MICINN-Spain/FEDER-UE, grant number PGC2018-094548-B-I00.

\section{ACKNOWLEDGMENTS}

We thank Amaia Iturrospe for technical support during SAXS experiments.

\section{REFERENCES}

1. Commission Recommendation 2011/696/EU. Available from: http://data.europa.eu/eli/reco/2011/696/oj. Accessed 2019 Jul 4.

2. Cornier J, Owen A, Kwade A, Van de Voorde M, editors. Pharmaceutical Nanotechnology: Innovation and Production. Weinheim (Germany): Wiley-VCH; 2016.

3. Sun J, Kormakov S, Liu Y, Huang Y, Wu D, Yang Z. Recent Progress in Metal-Based Nanoparticles Mediated Photodynamic Therapy. Molecules 2018;23(7):1704.

4. Dasari Shareena TP, McShan D, Dasmahapatra AK, Tchounwou PB. A Review on Graphene-Based Nanomaterials in Biomedical Applications and Risks in Environment and Health. Nano-Micro Lett. 2018;10(3):53.

5. Chen X, Liua Y, Ma Q. Recent advances in quantum dot-based electrochemiluminescence sensors. J Mater Chem C. 2018;6(5):942-59.

6. Pomposo JA, editor. Single-Chain Polymer Nanoparticles: Synthesis, Characterization, Simulations, and Applications. Weinheim (Germany): Wiley-VCH; 2017. 
7. Han B, Qu C, Park K, Konieczny SF, Korc M. Recapitulation of complex transport and action of drugs at the tumor microenvironment using tumor-microenvironment-on-chip. Cancer Lett. 2016;380(1):319-29.

8. Chen F, Ehlerding EB, Cai W. Theranostic Nanoparticles. J Nucl Med. 2014;55(12):1919-22.

9. Blanco E, Shen H, Ferrari M. Principles of nanoparticle design for overcoming biological barriers to drug delivery. Nat Biotechnol. 2015;33(9):941-51.

10. Champion JA, Walker A, Mitragotri S. Role of Particle Size in Phagocytosis of Polymeric Microspheres. Pharm Res. 2008;25(8):1815-21.

11. Decuzzi P, Ferrari M. The Receptor-Mediated Endocytosis of Nonspherical Particles. Biophys J. 2008;94(10): 3790-7.

12. Foroozandeh P, Aziz AA. Insight into Cellular Uptake and Intracellular Trafficking of Nanoparticles. Nanoscale Res Lett. 2018;13:339.

13. Mao X, Xu J, Cui $\mathrm{H}$. Functional Nanoparticles for Magnetic Resonance Imaging. Wiley Interdiscip Rev Nanomed Nanobiotechnol. 2016;8(6): 814-41.

14. Cormode DP, Naha PC, Fayad ZA. Nanoparticle Contrast Agents for Computed Tomography: A Focus on Micelles. Contrast Media Mol Imaging. 2014;9(1): 37-52.

15. Goel S, England CG, Chen F, Cai W. Positron Emission Tomography and Nanotechnology: A Dynamic Duo for Cancer Theranostics. Adv Drug Deliv Rev. 2017;113:157-76.

16. De-La-Cuesta J, González E, Pomposo JA. Advances in Fluorescent Single-Chain Nanoparticles. Molecules. 2017;22:1819.

17. Deng R, Lin D, Zhu L, Majumdar S, White JC, Gardea-Torresdey JL, Xing B. Nanoparticle interactions with co-existing contaminants: joint toxicity, bioaccumulation and risk. Nanotoxicology. 2017;11(5):591-612.

18. Teodorescu M, Bercea M. Poly(vinylpyrrolidone)—A Versatile Polymer for Biomedical and Beyond Medical Applications. Polym Plast Technol Eng. 2015;54(9): 923-43.

19. Latorre-Sanchez A, Pomposo JA. Recent bioinspired applications of single-chain nanoparticles. Polym Int. 2016;65(8):855-60.

20. Sanchez-Sanchez A, Akbari S, Moreno AJ, Lo Verso F, Arbe A, Colmenero J, Pomposo JA. Design and Preparation of Single-Chain Nanocarriers Mimicking Disordered Proteins for Combined Delivery of Dermal Bioactive Cargos. Macromol Rapid Commun. 2013;34: 1681-6.

21. Cheng CC, Lee DL, Liao ZS, Huang JJ. Stimuli-responsive single-chain polymeric nanoparticles towards the development of efficient drug delivery systems. Polym Chem. 2016;7:6164-9.

22. Kröger APP, Hamelmann NM, Juan A, Lindhoud S, Paulusse JMJ. Biocompatible Single-Chain Polymer Nanoparticles for Drug Delivery-A Dual Approach. ACS Appl Mater Interfaces. 2018;10(37):30946-51.

23. Kröger APP, Paulusse JMJ. Single-Chain Polymer Nanoparticles in Controlled Drug Delivery and Targeted Imaging. J Control Release. 2018;286:326-47. 
24. González-Burgos M, González E, Pomposo JA. Excellent Stability in Water of Single-Chain Nanoparticles against Chain Scission by Sonication. Macromol Rapid Commun. 2018;39:1700675.

25. Dasari S, Tchounwou PB. Cisplatin in cancer therapy: molecular mechanisms of action. Eur J Pharmacol. 2014;740:364-78.

26. Chae YK, Yousaf M, Malecek MK, Carneiro B, Chandra S, Kaplan J, Kalyan A, Sassano A, Platanias LC, Giles F. Statins as anti-cancer therapy: can we translate preclinical and epidemiologic data into clinical benefit? Discov Med. 2015;20:413-27.

27. Hong Y, Lam JWY, Tang BZ. Aggregation-induced emission: Phenomenon, mechanism and applications. Chem Commun. 2009;29:4332-53.

28. Liu Y, Deng C, Tang L, Qin A, Hu R, Sun JZ, Tang BZ. Specific Detection of D-Glucose by a Tetraphenylethene-Based Fluorescent Sensor. J Am Chem Soc. 2011;133(4):660-3.

29. Walling C. Fenton's reagent revisited. Acc Chem Res. 1975;8(4):125-31.

30. Zhu X, Lu P, Chen W, Dong J. Studies of UV crosslinked poly( $N$-vinylpyrrolidone) hydrogels by FTIR, Raman and solid-state NMR spectroscopies. Polymer. 2010;51(14):3054-63.

31. Pomposo JA, Perez-Baena I, Buruaga L, Alegría A, Moreno AJ, Colmenero J. On the Apparent SEC Molecular Weight and Polydispersity Reduction upon Intramolecular Collapse of Polydisperse Chains to Unimolecular Nanoparticles. Macromolecules. 2011;44(21):8644-9.

32. Arbe A, Pomposo JA, Moreno AJ, Lo Verso F, González-Burgos M, Asenjo-Sanz I, et al. Structure and dynamics of single-chain nano-particles in solution. Polymer. 2016;105:532-44.

33. Hammouda B. Small-Angle Scattering From Branched Polymers. Macromol Theory Simul. 2012;21(6):372-81.

34. Rubinstein M, Colby RH. Polymer Physics. Oxford (UK): Oxford University Press; 2003.

35. Gonzalez-Burgos M, Latorre-Sanchez A, Pomposo JA. Advances in single chain technology. Chem Soc Rev. 2015;44:6122-42.

36. Chinen AB, Guan CM, Ferrer JR, Barnaby SN, Merkel TJ, Mirkin CA. Nanoparticle Probes for the Detection of Cancer Biomarkers, Cells, and Tissues by Fluorescence. Chem Rev. 2015;115(19):10530-74.

37. Yang B, Zhang X, Zhang X, Huang Z, Wei Y, Tao L. Fabrication of aggregation-induced emission based fluorescent nanoparticles and their biological imaging application: recent progress and perspectives. Mater Today 2016;19(5):284-91.

38. Hong Y, Lam JWY, Tang BZ. Aggregation-induced emission. Chem Soc Rev. 2011;40(11):5361-88.

How to cite this article:

Asenjo-Sanz I, Del-Corte M, Pinacho-Olaciregui J, González-Burgos M, González E, Verde-Sesto E, Arbe A, Colmenero J, Pomposo JA. Preparation and Preliminary Evaluation of Povidone Single-Chain Nanoparticles as Potential Drug Delivery Nanocarriers. Med One. 2019;4:e190013. https://doi.org/10.20900/mo.20190013 